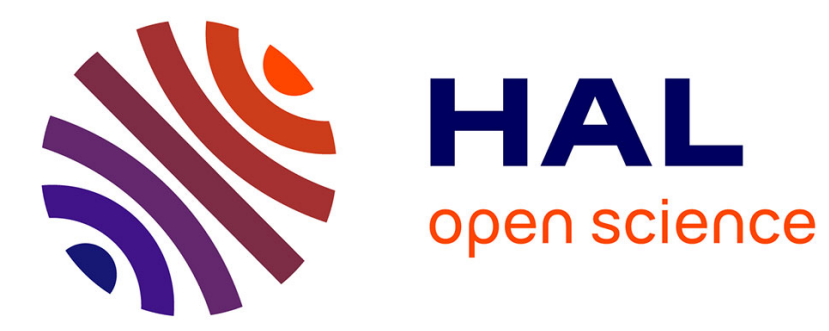

\title{
High-Gain Dead-Zone Observers for Linear and Nonlinear Plants
}

M. Cocetti, Sophie Tarbouriech, Luca Zaccarian

\section{To cite this version:}

M. Cocetti, Sophie Tarbouriech, Luca Zaccarian. High-Gain Dead-Zone Observers for Linear and Nonlinear Plants. IEEE Control Systems Letters, 2019, 3 (2), pp.356-361. 10.1109/LCSYS.2018.2880931 . hal-01970882

\section{HAL Id: hal-01970882 \\ https://hal.laas.fr/hal-01970882}

Submitted on 6 Jan 2019

HAL is a multi-disciplinary open access archive for the deposit and dissemination of scientific research documents, whether they are published or not. The documents may come from teaching and research institutions in France or abroad, or from public or private research centers.
L'archive ouverte pluridisciplinaire HAL, est destinée au dépôt et à la diffusion de documents scientifiques de niveau recherche, publiés ou non, émanant des établissements d'enseignement et de recherche français ou étrangers, des laboratoires publics ou privés. 


\title{
High-gain dead-zone observers for linear and nonlinear plants
}

\author{
M. Cocetti, S. Tarbouriech, L. Zaccarian
}

\begin{abstract}
We propose an adaptive dead-zone mechanism to robustify observers against high-frequency noise. The construction applies to Luenberger observers and high-gain observers for plants in strict feedback form. The dead-zone improves performances by trimming a portion of the output injection term and trapping the high frequency noise in the dead band. We show that the observer gain and the adaptation parameters can be obtained by solving a linear matrix inequality, whose feasibility only requires detectability of the plant. The parameters obtained through this optimization ensure (in the absence of noise) global exponential stability of the estimation error dynamics, and input-to-state stability (ISS) from the measurement noise to the estimation error.
\end{abstract}

\section{Index Terms}

Observers for Linear systems, Adaptive systems, Filtering, LMIs, Lyapunov methods.

\section{INTRODUCTION}

$\mathbf{R}$ OBUSTLY reconstructing the state of a plant from input-output measurements is one of the most fundamental problems in control theory. The first milestone has been obtained by David Luenberger in [1] and [2], where the problem of state estimation for linear plants has been solved by means of a dynamic filter, today called Luenberger observer. The need for robust observation laws motivated nonlinear extensions of the linear loop transfer recovery approach [3] leading to high-gain solutions that appeared in the early nineties [4], [5], and later became popular tools solving nonlinear control problems ranging from output stabilization to tracking and regulation. This great impact was also possible thanks to the output stabilization techniques developed in [6] and to the semiglobal separation principle obtained in [7], [8]. These results allow designing feedback laws as if the state were available and then use an estimate provided by a sufficiently fast observer. The prices to pay for this flexibility are essentially twofold: first, peaking phenomena, and second high sensitivity to measurements noise. The latter was already well known in the framework of linear observers [9] and entails the classical trade-off between bandwidth and noise rejection.

Despite this high sensitivity, high-gain observers are ISS from the measurement noise to the estimation error [10], [11], [12]. To reduce peaking and noise sensitivity, observation schemes with improved rejection may comprise a bank of different observers (a multi-observer) as in [13], where each observer has a different sensitivity to noise and convergence speed. Then, a supervisor chooses which observer is providing the best estimate at any given time. See also [14] where robustness with respect to an uncertain plant is studied (each observer is designed for a different value of the uncertain parameter). Alternatively, one may use an aggressive observer gain for transient estimation and a more relaxed one for steady-state performance [15]. However, there are many design challenges in using this approach, e.g., it is hard to properly select the switching time/mechanism (like trigger or threshold based mechanism) and the intrinsic discontinuous behavior of the observer is dangerous when combined with phenomena as peaking. A similar, but simpler, approach has been also proposed in [16] where a piecewise linear gain function is used. The limiting case of arbitrarily fast switching among different observers or different gains can be thought of as continuous adaptation, as in [17], [18], [19], where the observer gain is dynamically adapted according to the difference $(\hat{y}-y)$.

Nonlinear, time-varying or adaptive output injection/correction terms can also be considered, as in [20], [21], [22], to obtain superior performance or to deal with nonlinear plants that do not have special normal forms or do not satisfy Lipschitz-like conditions. Finally, recently the so-called low-power approach has been proposed [23], [24], which increases the dimension of the observer state but reduces the sensitivity to noise and the peaking phenomena.

In this work we propose here to apply an artificial dead-zone to the output injection term $(\hat{y}-y)$. The resulting "deadzonated" observer better rejects high-frequency noise by cutting the part of it falling inside the dead-band. The dead-band amplitude is dynamically adapted according to the noise level, providing another useful information.

The dead-zone has a destabilizing effect on the estimation error dynamics, indeed in a ball around the zero estimation error the observer runs in open loop. However if the adaptation mechanism is carefully designed, the dead-zone rapidly converges to the identity function and in the absence of noise retrieves the classical output injection term $(\hat{y}-y)$. We show here that

M. Cocetti is with the Department of Industrial Engineering, 9 Sommarive, University of Trento, Trento, IT, 38123. Email: (matteo.cocetti, luca.zaccarian) dunitn.com and also with LAAS-CNRS, University of Toulouse, CNRS, Toulouse, France.

S. Tarbouriech and L. Zaccarian are with LAAS-CNRS, University of Toulouse, CNRS Toulouse, France. Email: (tarbour, luca.zaccarian) @laas.fr

This research was supported by grant PowerLyap funded by CaRiTRo and DRTS Transmission Systems, and in part by ANR grant HANDY. 
both the synthesis of the observer gain and of the adaptation mechanism can be cast as Linear Matrix Inequality (LMI), whose feasibility only requires detectability of the plant.

The use of the dead-zone is motivated by results developed for linear systems subject to sector bounded nonlinearities, such as global and local sector conditions [25], [26]. These tools have been extensively used in combination with LMIs in the context of anti-windup design, but rarely employed for observers, even if there are a few remarkable exceptions [27], [28], [22]. Our solution is indeed somewhat inspired by [28], where similar adaptations are adopted to preserve stability in the presence of a saturated (rather than dead-zonated) output injection term, geared towards efficiently dealing with measurement outliers.

A preliminary version of this work has been presented in [29]. The new contributions are threefold. First, we show that the convergence rate for dead-zone observer is exponential and not only asymptotic. Second, the dead-zone adaptation mechanism is now decentralized, in the sense that each channel of the output injection term can have a different dead-zone amplitude. This new property may be very useful if one channel is noisier than the other. Third, we show that the proposed dead-zone output injection can be successfully applied also to nonlinear high-gain observers designed for plants in strict feedback form.

The paper is organized as follows. Section II formulates the problem for Linear Time Invariant (LTI) plants. Section III contains the main result showing Global Exponential Stability (GES) of the error dynamics in the absence of noise and Input to State Stability (ISS) in the presence of measurement noise. Section IV presents extensions of the dead-zone construction to high-gain observers. An example is provided in Section V. Finally, conclusions are offered in Section VI.

Notation: $\mathbb{R}^{n}$ denotes the set of real vectors of dimension $n$. Given a constant $c \in \mathbb{R}, \mathbb{R}_{\geq c}:=[c, \infty)$. Given two vectors $x \in \mathbb{R}^{n}, y \in \mathbb{R}^{m},(x, y):=\left[x^{\top} y^{\top}\right]^{\top} \in \mathbb{R}^{n+m}$. For a matrix $M, M^{\top}$ denotes its transpose and $M^{-1}$ its inverse (if it exists). $M>0(M \geq 0)$ denotes positive definiteness (semi-definiteness) and $\operatorname{He}(M):=\left(M+M^{\top}\right) . \operatorname{Diag}_{>0}^{n}\left(\operatorname{resp} \operatorname{Diag}_{\geq 0}^{n}\right)$ denotes the set of diagonal positive (resp. semi) definite matrices of dimension $n \times n$, and $\operatorname{Sym}_{>0}^{n}\left(\mathrm{resp} \mathrm{Sym}_{>0}^{n}\right)$ the set of symmetric positive (resp. semi) definite matrices of dimension $n \times n$. The vector $\mathbf{1}$ is $\mathbf{1}:=(1,1, \ldots, 1) \in \mathbb{R}^{p}$. The symmetric scalar saturation function with levels $\pm b \in \mathbb{R}_{>0}$, is $\operatorname{defined}_{\text {as }} \operatorname{sat}_{b}(a):=\min \{b, \max \{a,-b\}\}$. The dead-zone function is $\mathrm{dz}_{b}(a):=a-\operatorname{sat}_{b}(a)$. In this paper we consider constrained nonlinear systems of the form

$$
x \in C, \quad \dot{x}=f(x, u),
$$

where $C \subset \mathbb{R}^{n}$ is a closed set, and $f$ is a continuous function such that $0=f(0,0)$.

For $u \equiv 0$ the origin of (1) is Globally Exponentially Stable (GES), if for any initial condition $\phi(0) \in C$ solutions satisfy $|\phi(t)| \leq k \exp (-\alpha t) \phi(0)$ for some positive constants $k, \alpha>0$. System (1) is Input to State Stable (ISS) if there exist a class $\mathcal{K} \mathcal{L}$ function $\beta$ and a class $\mathcal{K}$ function $\gamma$ such that for any initial condition $\phi(0) \in C$ solutions to (1) satisfy $|\phi(t)| \leq \beta(|\phi(0)|, t)+\gamma\left(\sup _{0 \leq \tau \leq t}|u(\tau)|\right)$ for all $t \in \operatorname{dom}(\phi)$.

\section{LTI PROBLEM FORMULATION}

In this paper we consider a continuous-time LTI system ("the plant") of the following form,

$$
\left\{\begin{array}{l}
\dot{x}=A x+B u \\
y=C x+D u+v,
\end{array}\right.
$$

where $x \in \mathbb{R}^{n}$ is the state, $u \in \mathbb{R}^{m}$ is the input, $y \in \mathbb{R}^{p}$ is the measured output and $v \in \mathbb{R}^{p}$ is a measurement noise. Following the preliminary work in [29], for dealing with high-frequency noise affecting the plant output, we introduce a Luenberger observer whose output injection is "dead-zonated" as follows

$$
\left\{\begin{array}{l}
\dot{\hat{x}}=A \hat{x}+B u+L \mathrm{dz}_{\sqrt{\sigma}}(\hat{y}-y) \\
\hat{y}=C \hat{x}+D u,
\end{array}\right.
$$

where $\hat{x} \in \mathbb{R}^{n}$ is the estimated state, $\hat{y} \in \mathbb{R}^{p}$ is the estimated output and $\sigma \in \mathbb{R}_{\geq 0}^{p}$ is a vector whose entries are non-negative and define the amplitude of the dead-zone on the corresponding output channel. THe matrix $L \in \mathbb{R}^{n \times p}$ is the classical observer gain. The function $\mathrm{dz}_{\sqrt{\sigma}}: \mathbb{R}^{p} \rightarrow \mathbb{R}^{p}$ is a decentralized vector-valued dead-zone defined as follows

$$
\mathrm{dz}_{\sqrt{\sigma}}(y):=\left[\begin{array}{lll}
\mathrm{dz}_{\sqrt{\sigma_{1}}}\left(y_{1}\right) & \ldots & \mathrm{dz}_{\sqrt{\sigma_{p}}}\left(y_{p}\right)
\end{array}\right]^{\top},
$$

where $\sqrt{\sigma}:=\left(\sqrt{\sigma_{1}}, \ldots, \sqrt{\sigma_{p}}\right) \in \mathbb{R}^{p}$ is a component-wise square root. For $\sigma$ we propose the following adaptation law

$$
\dot{\sigma}=-\Lambda \sigma+\left[\begin{array}{c}
(\hat{y}-y)^{\top} R_{1}(\hat{y}-y) \\
\vdots \\
(\hat{y}-y)^{\top} R_{p}(\hat{y}-y)
\end{array}\right], \quad \sigma \in \mathbb{R}_{\geq 0}^{p}
$$

where $\Lambda \in \operatorname{Diag}_{>0}^{p}$ is a diagonal positive definite matrix, and $R_{1}, \ldots, R_{p} \in \mathrm{Sym}_{\geq 0}^{p}$ are symmetric positive semi-definite matrices. The constraint $\sigma \in \mathbb{R}_{>0}^{p}$ means that $\sigma$ belongs to the closed $p$-dimensional positive orthant, which is an invariant set for (5). It is worth to notice that non-negativity of $\sigma$ makes the square root $\sqrt{\sigma}$ always well defined. 
The idea behind observer (3) is that the dead-zone provides a zero output correction term around $(\hat{y}-y)=0$ so that high frequency noise is filtered out. However, the dead-zone also has a destabilizing effect on the error dynamics so that a fast enough adaptation of $\sigma$ is necessary to ensure convergence to zero. Indeed, for a fixed dead-zone amplitude $\sqrt{\sigma}$, signals $\hat{y}$ and $y$ which are close enough would never synchronize. The adaptive mechanism (5) is designed to weigh two antagonistic effects: first, the "adaptation speed" selected by $\Lambda$, and second, the "filtering action" tuned by $R_{1}, \ldots, R_{p}$. Intuitively speaking, selecting $\Lambda$ large enough, and $R_{1}, \ldots, R_{p}$ sufficiently small we can recover a classical Luenberger observer. On the other hand, setting $\Lambda$ small and $R_{1}, \ldots, R_{p}$ large we slow down the convergence rate while increasing the filtering capability.

Remark 1: The initial condition $\sigma(0)$ for (5) can be taken small or zero if we expect measurement with a low noise level. On the contrary, if large amplitude noise is expected, then larger values of $\sigma(0)$ may lead to improved transient responses. $\lrcorner$

\section{MAIN RESULTS}

In this section we prove a few good properties for the dead-zone observers in (3). We show that for a detectable plant, the parameters $\Lambda$ and $R_{i}$, can always be designed to obtain GES of the error dynamics in the absence of measurement noise, and ISS from the measurement noise to the estimation error.

\section{A. Global Exponential Stability (GES)}

Given (3) and the adaptation law (5) we cast the synthesis of the parameters $L, \Lambda, R_{1}, \ldots, R_{p}$ as an LMI problem. In the absence of noise, this tuning procedure ensures GES of the estimation error dynamics and of the adaptive dynamics $\sigma$. To this end, define the estimation error as $e=\hat{x}-x$ and the vector-valued saturation function as $\operatorname{sat}_{\sqrt{\sigma}}(y):=y-\mathrm{dz}_{\sqrt{\sigma}}(y)$, where the vector-valued dead-zone is defined in (4). Then, after few manipulations, we obtain the following representation for the error arising from (3), (5),

$$
\begin{aligned}
& \dot{e}=(A+L C) e-L v-L \mathrm{sat}_{\sqrt{\sigma}}(C e-v) \\
& \dot{\sigma}=-\Lambda \sigma+\left[\begin{array}{c}
(C e-v)^{\top} R_{1}(C e-v) \\
\vdots \\
(C e-v)^{\top} R_{p}(C e-v)
\end{array}\right] \quad \sigma \in \mathbb{R}_{\geq 0}^{p} .
\end{aligned}
$$

Equation (6) represents the error dynamics of a classical Luenberger observer plus a perturbation term whose amplitude is ruled by $\sqrt{\sigma}$. What follows is the main result of this paper for the LTI case, namely for the class of systems (2).

Theorem 1: Consider the following LMI in the optimization variables $P \in \operatorname{Sym}_{>0}^{n}, X \in \mathbb{R}^{n \times p}, \Lambda \in \operatorname{Diag}_{>0}^{p}, R \in \operatorname{Sym}_{\geq 0}^{p}$, $U \in \operatorname{Diag}_{\geq 0}^{p}$,

$$
\operatorname{He}\left[\begin{array}{cc}
P A+X C+C^{\top} R C & -X \\
U C & -U-\Lambda
\end{array}\right]<0 .
$$

Any feasible solution to (7), together with the choice

$$
L:=P^{-1} X, \quad R:=\sum_{j=1}^{p} R_{j}
$$

makes (6) globally exponentially stable to the origin for $v=0$ and ISS from $v$ to $(e, \sqrt{\sigma})$.

Remark 2: The adaptation law in (5) allows for a completely decentralized form where the amplitude of each dead-zone is adapted independently. This can be done by solving (7) for a diagonal $R$ and then associating each diagonal element $r_{i i}$ to the corresponding $\sigma_{i}$ dynamics. The resulting adaptation law yields $\dot{\sigma}_{i}=-\lambda_{i i} \sigma_{i}+r_{i i}\left(\hat{y}_{i}-y_{i}\right)^{2}$.

Proof. Strict negativity of (7) implies that there exists a sufficiently small $c_{0} \in \mathbb{R}_{>0}$ such that,

$$
H:=\mathrm{He}\left[\begin{array}{cc}
P A+X C+C^{\top} R C & -X \\
U C & \left(c_{0}-1\right) \Lambda-U
\end{array}\right] \leq-2 c_{0} I
$$

Then, let us consider the candidate Lyapunov function $V(e, \sigma):=e^{\top} P e+2 \mathbf{1}^{\top} \sigma$, where $P \in \operatorname{Sym}_{>0}^{n}$. The function $V(e, \sigma)$ is positive definite and radially unbounded on $\mathbb{R}^{n} \times \mathbb{R}_{\geq 0}^{p}$ and satisfies the following bounds,

$$
\alpha_{1}|(e, \sqrt{\sigma})|^{2} \leq V(e, \sigma) \leq \alpha_{2}|(e, \sqrt{\sigma})|^{2},
$$

where $\alpha_{1}:=\min \left\{\lambda_{\min }(P), 2\right\}$, and $\alpha_{2}:=\max \left\{\lambda_{\max }(P), 2\right\}$, so that the Lie derivative along the flow of (6) yields

$$
\begin{aligned}
\dot{V}(e, \sigma)= & e^{\top}(A+L C)^{\top} P e+e^{\top} P(A+L C) e \\
& -2 e^{\top} P L \operatorname{sat}_{\sqrt{\sigma}}(C e-v)-2 v^{\top} L^{\top} P e-2 \mathbf{1}^{\top} \Lambda \sigma \\
& +2 \sum_{j=1}^{p}(C e-v)^{\top} R_{j}(C e-v) .
\end{aligned}
$$


To enforce (strict) negativity of (11) we first consider a global sector condition for cone bounded nonlinearities [25] and then use the fact that the saturation levels are proportional to $\sqrt{\sigma}$ and thus $\operatorname{sat}_{\sqrt{\sigma}}^{\top}(C e)$ sat $\sqrt{\sigma}(C e)$ never exceeds $\mathbf{1}^{\top} \sigma$. These observations translate into the following inequalities

$$
\begin{aligned}
\operatorname{sat}_{\sqrt{\sigma}}^{\top}(C e-v) U\left(C e-v-\operatorname{sat}_{\sqrt{\sigma}}(C e-v)\right) & \geq 0, \\
\mathbf{1}^{\top} \Lambda \sigma-\operatorname{sat}_{\sqrt{\sigma}}^{\top}(C e-v) \Lambda \operatorname{sat}_{\sqrt{\sigma}}(C e-v) & \geq 0,
\end{aligned}
$$

for any matrices $U \in \operatorname{Diag}_{>0}^{p}, \Lambda \in \operatorname{Diag}_{>0}^{p}$. Multiplying (12a) by two and (12b) by $2\left(1-c_{0}\right)$, and adding them to (11), we obtain the following upper-bound, which uses $X=P L$ and $R:=\sum_{j=1}^{p} R_{j}$,

$$
\begin{aligned}
\dot{V}(e, \sigma) \leq & {\left[\begin{array}{c}
e \\
\operatorname{sat}_{\sqrt{\sigma}}(C e-v)
\end{array}\right]^{\top} H\left[\begin{array}{c}
e \\
\operatorname{sat}_{\sqrt{\sigma}}(C e-v)
\end{array}\right] } \\
& -2 c_{0} \mathbf{1}^{\top} \Lambda \sigma-2 v^{\top} L^{\top} P e+2 v^{\top} R v \\
& -4 v^{\top} R C e-2 \operatorname{sat}_{\sqrt{\sigma}}^{\top}(C e-v) U v .
\end{aligned}
$$

To prove GES in the absence of disturbances, let us set $v=0$ and plug (9) into (13) to obtain the following inequality

$$
\begin{aligned}
\dot{V}(e, \sigma) & \leq-2 c_{0}\left|\left(e, \operatorname{sat}_{\sqrt{\sigma}}(C e)\right)\right|^{2}-2 c_{0} \mathbf{1}^{\top} \Lambda \sigma \\
& \leq-2 c_{1}|(e, \sqrt{\sigma})|^{2} \leq-\frac{2 c_{1}}{\alpha_{2}} V(e, \sigma),
\end{aligned}
$$

where we used the rightmost bound in (10) and $c_{1}:=c_{0} \min \left\{1, \lambda_{\min }(\Lambda)\right\}$. Applying the comparison lemma and using the leftmost bound in (10) we immediately obtain $|(e, \sqrt{\sigma})| \leq \sqrt{\alpha_{2} / \alpha_{1}} \exp \left(-c_{1} / \alpha_{2}\right)\left|\left(e_{0}, \sqrt{\sigma_{0}}\right)\right|$, which proves global exponential stability for $(e, \sqrt{\sigma})$. The exponential bound can then be easily extended to $(e, \sigma)$, see [28].

To prove the ISS statement, let us define $c_{2}:=|R|, c_{3}:=\left|P L+2 C^{\top} R\right|, c_{4}:=|U|$, so that (13) can be compactly rewritten as

$$
\begin{aligned}
\dot{V}(e, \sigma) \leq & -2 c_{1}|(e, \sqrt{\sigma})|^{2}+2 c_{2}|v|^{2} \\
& +2 c_{3}|e||v|+2 c_{4}|\sqrt{\sigma}||v| \\
\leq & -c_{1}|(e, \sqrt{\sigma})|^{2}+c_{1}^{-1}\left(2 c_{1} c_{2}+c_{3}^{2}+c_{4}^{2}\right)|v|^{2} \\
\leq & -c_{1}|(e, \sqrt{\sigma})|^{2}<0,
\end{aligned}
$$

which holds for all $|(e, \sqrt{\sigma})|^{2}>c_{1}^{-2}\left(2 c_{1} c_{2}+c_{4}^{2}+c_{3}^{2}\right)|v|^{2}:=c_{5}^{2}|v|^{2}$ and we conclude that (6) is ISS from $v$ to $(e, \sqrt{\sigma})$ with an ISS gain proportional to $c_{5}$. In (14) we used the Young inequalities $2|e||v| \leq \frac{c_{1}}{c_{3}}|e|^{2}+\frac{c_{3}}{c_{1}}|v|^{2}$ and $2|v||\sqrt{\sigma}| \leq \frac{c_{1}}{c_{4}}|\sqrt{\sigma}|^{2}+\frac{c_{4}}{c_{1}}|v|^{2}$.

Theorem 1 ensures exponential convergence of the estimation error in the absence of disturbances, and its boundedness in the presence of bounded disturbances.

This is desirable for nonlinear observers, because in a nonlinear setting diverging solutions may arise with arbitrarily small measurement noise [30]. Moreover the ISS property ensures graceful performance degradation, another desirable property for (6).

\section{B. Feasibility}

Theorem 1 provides only a sufficient condition to enforce GES, however feasibility of (7) can be exactly characterized.

Proposition 1: The LMI (7) is feasible if and only if pair $(C, A)$ is detectable.

Proof. Necessity follows from standard detectability results. Indeed, if pair $(C, A)$ is not detectable, no asymptotic observer exists and thus (7) must be infeasible from Theorem 1. To prove sufficiency we use [31, Thm 16.6] to get that detectability of $(C, A)$ implies the existence of a matrix $P \in \operatorname{Sym}_{>0}^{n}$ satisfying

$$
A^{\top} P+P A-C^{\top} C<0 .
$$

Consider (7) and select $X=-3 C^{\top}, \Lambda=U=I$ and $R=I / 2$. Pre/post multiplying by the congruence transformation matrix $\left[\begin{array}{cc}I & C^{\top} \\ 0 & I\end{array}\right]$, yields $\left[\begin{array}{cc}A^{\top} P+P A-C^{\top} C & 0 \\ 0 & -4 I\end{array}\right]<0$, which is feasible thanks to (15).

Remark 3: Under the detectability assumption, condition (7) is always feasible even when a stabilizing gain $L$ is a priori fixed. In this case we can think of (3) as a dead-zone augmentation, i.e., an adaptive output injection mechanism enhancing the performance of a pre-designed observer. The LMI in (7) can still be used as a design tool fixing a stabilizing $L$ (i.e., such that $(A+L C)$ is Hurwitz) and replacing $X$ by $P L$. The arising LMI is always feasible and provides a convenient way to design the adaptation parameters $\Lambda$ and $R$. 


\section{HIGH-GAIN DEAD-ZONE OBSERVERS FOR NONLINEAR PLANTS}

In this section we extend the dead-zone design to multi-input, single-output nonlinear plant in the following strict feedback form

$$
\left\{\begin{aligned}
\dot{x}_{1} & =x_{2}+f_{1}\left(x_{1}, u\right) \\
& \vdots \\
\dot{x}_{n-1} & =x_{n}+f_{n-1}\left(x_{1 \ldots n-1}, u\right) \\
\dot{x}_{n} & =f_{n}\left(x_{1 \ldots n}, u\right) \\
y & =x_{1}+v,
\end{aligned}\right.
$$

where $x:=\left(x_{1}, \ldots, x_{n}\right) \in \mathbb{R}^{n}$ is the state, $u \in \mathbb{R}^{m}$ is the input, $y \in \mathbb{R}$ is the output. We use the shorthand notation $x_{1 \ldots i}:=\left(x_{1}, \ldots, x_{i}\right) \in \mathbb{R}^{i}$ to stress that $f_{i}$ depends only on the first $i$ components of the state vector $x$. The functions $f_{i}$ are assumed to be continuous. Conditions for the existence of a local or global diffeomorphism that maps a generic nonlinear system into the strict feedback form (16) are analyzed in [32, Thm 4.1] and are related to the existence of uniform full relative degree. For the sake of compactness we rewrite (16) as follows

$$
\left\{\begin{array}{l}
\dot{x}=A x+f(x, u) \\
y=C x+v
\end{array}\right.
$$

where pair $(C, A)$ is in prime form, therefore observable, and $f: \mathbb{R}^{n} \times \mathbb{R}^{m} \rightarrow \mathbb{R}^{n}$ is a continuous vector-valued map obtained stacking the functions $f_{i}$ for $i=1, \ldots, n$. Following the construction of Section II consider the following high-gain dead-zone observer for (17)

$$
\left\{\begin{aligned}
\dot{\hat{x}} & =A \hat{x}+\hat{f}(\hat{x}, u)+\mathscr{E}^{-1}(\epsilon) L \mathrm{dz}_{\sqrt{\sigma}}(\hat{y}-y) \\
\hat{y} & =C \hat{x} \\
\epsilon \dot{\sigma} & =-\Lambda \sigma+R(\hat{y}-y)^{2},
\end{aligned}\right.
$$

where $\hat{x} \in \mathbb{R}^{n}$ is the estimated state, $\hat{y} \in \mathbb{R}$ is the estimated output, and $\hat{f}_{i}$ are some approximations of functions $f_{i}$. The vector $L \in \mathbb{R}^{n}$ is the observer gain and matrix $\mathscr{E}(\epsilon):=\operatorname{diag}\left(\epsilon, \ldots, \epsilon^{n}\right) \in \mathbb{R}^{n \times n}$ contains the powers of the high-gain parameter $\epsilon \in \mathbb{R}_{(0,1)}$, which is assumed to be small. For the analysis that follows it is convenient to define a re-scaled version of the error coordinates as follows

$$
e=\epsilon^{-1} \mathscr{E}(\epsilon)(\hat{x}-x)
$$

so that combining (18) and (17) the error dynamics results

$$
\left\{\begin{aligned}
\epsilon \dot{e}= & (A+L C) e+\mathscr{E}(\epsilon)(\hat{f}(\hat{x}, u)-f(x, u)) \\
& -L v-L \text { sat }_{\sqrt{\sigma}}(C e-v) \\
\epsilon \dot{\sigma}= & -\Lambda \sigma+R(C e-v)^{2} .
\end{aligned}\right.
$$

From the upper-triangular structure of $\hat{f}-f$, we may select $\epsilon$ sufficiently small to dominate this mismatch and also speed up the observer dynamics. We ensure global convergence of the error by assuming the following [33].

Assumption 1: The difference $\hat{f}_{i}-f_{i}$ satisfies globally the Lipschitz-like condition

$$
\left|\hat{f}_{i}\left(\hat{x}_{1 \ldots i}, u\right)-f_{i}\left(x_{1 \ldots i}, u\right)\right| \leq \mu_{i}^{\circ}+\mu_{i} \sum_{j=1}^{i}\left|\hat{x}_{j}-x_{j}\right|,
$$

for all $x_{1 \ldots i} \in \mathbb{R}^{i}$ with $\mu_{i}^{\circ}, \mu_{i}$ independent of $u$.

From the structure of (21) and (19), follows that

$$
\begin{aligned}
|\mathscr{E}(\epsilon)(\hat{f}(\hat{x}, u)-f(x, u))| & \leq \epsilon \sum_{i=1}^{n} \epsilon^{i-1}\left(\mu_{i}^{\circ}+\mu_{i} \sum_{j=1}^{i} \epsilon^{1-j}\left|e_{j}\right|\right) \\
& \leq \epsilon \sum_{i=1}^{n} \epsilon^{i-1} \mu_{i}^{\circ}+\epsilon \sum_{i=1}^{n} \mu_{i}|e| \\
& \leq \epsilon \mu^{\circ}+\epsilon \mu|e|
\end{aligned}
$$

where we defined $\mu^{\circ}:=\sum_{i=1}^{n} \mu_{i}^{\circ}$ and $\mu:=\sum_{i=1}^{n} \mu_{i}$ and we used the fact that $\epsilon \in \mathbb{R}_{(0,1)}$. This upper bound will be useful in the proof of Theorem 2 .

Remark 4: As usually done in the high-gain observers literature, the global Lipschitz property in Assumption 1 can be relaxed to local Lipschitz if we assume that the state of the plant evolves inside a known compact set. If this is the case, we 
can saturate functions $\hat{f}_{i}$ outside such a compact set, and still obtain convergence for global $\hat{x}(0)$ provided $x(0)$ belongs to the compact set.

Remark 5: Global observers for nonlinear systems that do not satisfy global Lipschitz conditions can be still constructed using homogenization tools, see for example [20], [34].

Theorem 2: Suppose that $L, \Lambda, R$ have been designed according to (7) and that Assumption 1 holds, then (20) is ISS from $\left(v, \mu^{\circ}\right)$ to $(e, \sqrt{\sigma})$ and for $\mu^{\circ}=0, v=0$ the origin is GES for (20).

Proof. We follow similar steps as in Theorem 1. Consider a scaled candidate Lyapunov function $V(e, \sigma):=\epsilon e^{\top} P e+2 \epsilon \sigma$ so that the Lie derivative along the flow of (20) yields,

$$
\begin{aligned}
\dot{V}(e, \sigma) \leq & -c_{1}|(e, \sqrt{\sigma})|^{2}+c_{5}|v|^{2} \\
& +2 e^{\top} P \mathscr{E}(\epsilon)(\hat{f}(\hat{x}, u)-f(x, u)) \\
\leq & -c_{1}|(e, \sqrt{\sigma})|^{2}+c_{5}|v|^{2} \\
& +2|e||P \| \mathscr{E}(\epsilon)(\hat{f}(\hat{x}, u)-f(x, u))|,
\end{aligned}
$$

where $c_{1}$ and $c_{5}$ are defined in the proof of Theorem 1. Plugging (19) into (21) and using (22) we can upper-bound equation (23) as follows

$$
\begin{aligned}
\dot{V}(e, \sigma) \leq & -c_{1}|(e, \sqrt{\sigma})|^{2}+c_{5}|v|^{2}+2 \epsilon \mu^{\circ}|P||e|+\left.2 \epsilon \mu|P \||\right|^{2} \\
\leq & -\left(c_{1}-2 \epsilon \mu|P|\right)|(e, \sqrt{\sigma})|^{2}+c_{5}|v|^{2}+2 \epsilon \mu^{\circ}|P \|| e \mid \\
\leq & -\left(c_{1}-2 \epsilon \mu|P|-\epsilon c_{1}\right)|(e, \sqrt{\sigma})|^{2}+c_{5}|v|^{2} \\
& +\epsilon c_{1}^{-1}|P|^{2}\left(\mu^{\circ}\right)^{2}
\end{aligned}
$$

so that, for $\epsilon$ sufficiently small, system (20) is ISS in the sense defined in Theorem 2 and for $\mu^{\circ}=0, v=0$, GES.

\section{AN EXAMPLE}

We propose an example that compares a high-gain observer with and without the dead-zone mechanism proposed in Section IV. We show through simulations that the dead-zone mechanism successfully improves the noise rejection capability. Let us consider the Van der Pol oscillator,

$$
\left\{\begin{aligned}
\dot{x}_{1} & =x_{2} \\
\dot{x}_{2} & =-x_{1}+\gamma\left(1-x_{1}^{2}\right) x_{2}+u \\
y & =x_{1}+v
\end{aligned}\right.
$$

where $\gamma=5$. Equation (24) is in strict feedback form, but does not satisfy Assumption 1. However, assuming $u$ bounded the solutions to (24) evolve in a compact set and according to Remark 4 we can saturate the nonlinear part of the observer obtaining global practical convergence for global $\hat{x}(0)$ provided $x(0)$ belongs to a compact set. We propose the following dead-zonated high-gain observer

$$
\begin{aligned}
& \dot{\hat{x}}_{1}=\hat{x}_{2}+\epsilon^{-1} \ell_{1} \mathrm{dz}_{\sqrt{\sigma}}\left(\hat{x}_{1}-y\right) \\
& \dot{\hat{x}}_{2}=\operatorname{sat}_{M}\left(-\hat{x}_{1}+\hat{\gamma}\left(1-\hat{x}_{1}^{2}\right) \hat{x}_{2}+u\right)+\epsilon^{-2} \ell_{2} \mathrm{dz}_{\sqrt{\sigma}}\left(\hat{x}_{1}-y\right) \\
& \epsilon \dot{\sigma}=-\Lambda \sigma+R\left(\hat{x}_{1}-y\right)^{2},
\end{aligned}
$$

where $\hat{\gamma}=7$ and $M=20$. Parameters $L:=\left[\ell_{1}, \ell_{2}\right]^{\top} \in \mathbb{R}^{2}, \Lambda \in \mathbb{R}_{>0}, R \in \mathbb{R}_{\geq 0}$ have been designed according to the following convex optimization problem that maximizes the effect of the noise on the adaptation dynamics $\sigma$,

$$
\begin{aligned}
& \sup _{P, X, \Lambda, R} \operatorname{tr}(R) \text { subject to: } \\
& \operatorname{He}\left[\begin{array}{cc}
P A+X C+C^{\top} R C & -X \\
U C & -U-\Lambda
\end{array}\right]<0 \\
& \mathrm{He}\left[P A+X C+\alpha_{\min } P\right]<0 \\
& \operatorname{He}\left[P A+X C+\alpha_{\max } P\right]>0 \\
& 0<\Lambda \leq \Lambda_{\max }, R=R^{\top} \geq 0, P=P^{\top}>I .
\end{aligned}
$$

In addition to the LMI condition (7) we impose an interval for the possible convergence rates, so that $-\alpha_{\max } \leq \operatorname{Re}\left(\lambda_{k}(A+\right.$ $L C)) \leq-\alpha_{\min }$ where $\alpha_{\min }=1, \alpha_{\max }=100 \in \mathbb{R}_{>0}$ for this specific example. Finally, we select $\Lambda_{\max }=10 \in \mathbb{R}_{>0}$, to avoid an excessive time scale separation among the observer and the adaptation dynamics. Solving (25) we obtain the following values $L=\left[\begin{array}{ll}-68.36 & -68.06\end{array}\right]^{\top}, \Lambda=9.98$, and $R=7.51$. The high-gain parameter has been selected as $\epsilon=0.1$. 


\begin{tabular}{lrrrrrr}
\hline$R$ & 1.9 & 3.0 & 4.1 & 5.3 & 6.4 & 7.5 \\
\hline ENR High-gain & 32.6 & 32.6 & 32.6 & 32.6 & 32.6 & 32.6 \\
\hline ENR Dead-zone & 19.2 & 16.1 & 13.7 & 11.8 & 10.2 & 8.9 \\
\hline \multicolumn{7}{c}{ Table 1}
\end{tabular}
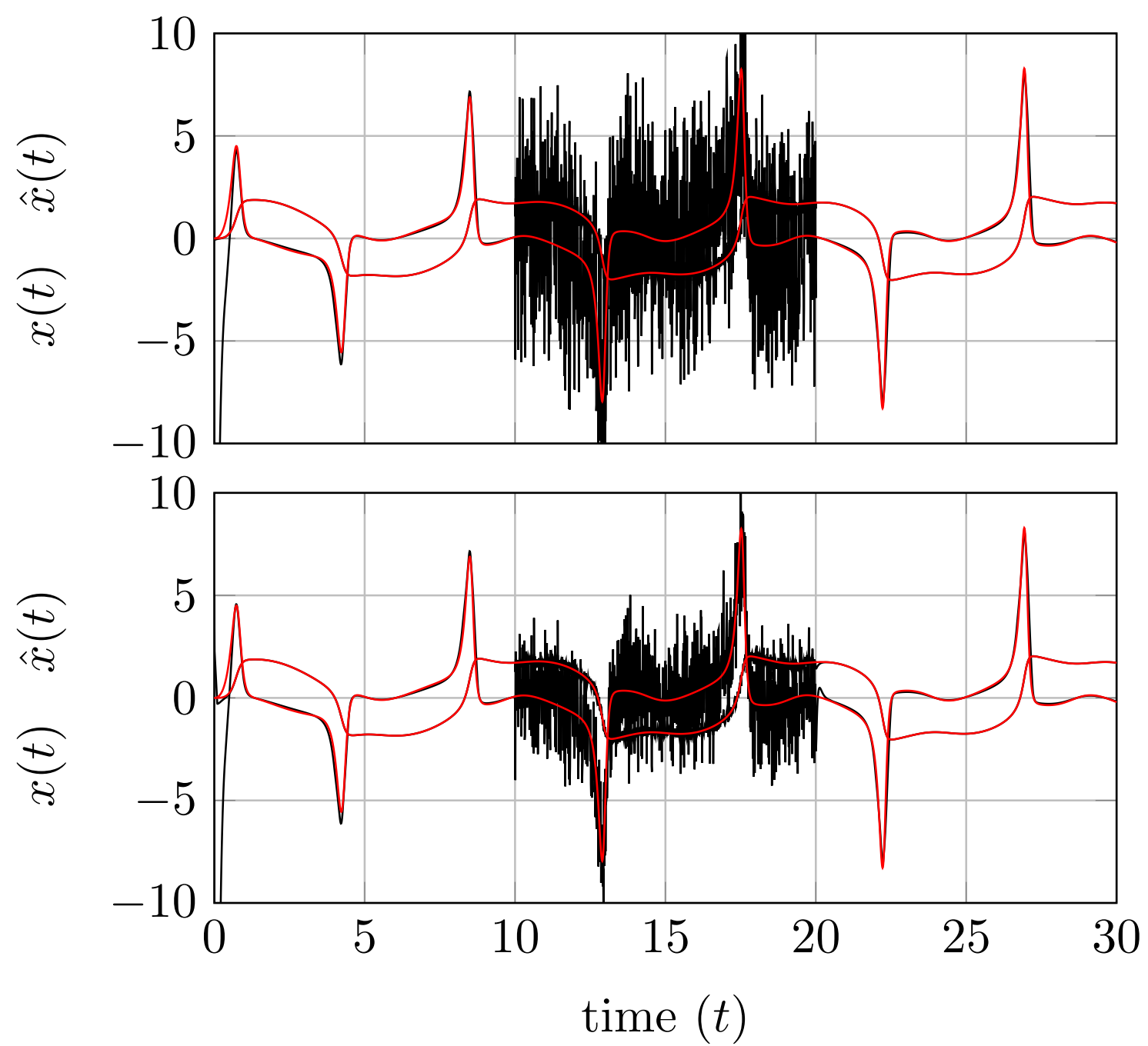

Figure 1. Estimated (black) and true (red) state with the bare high-gain observer (above) and the dead-zonated high-gain Observer (below) $(R=7.5)$. Notice the significant estimation error reduction in the lower trace.

Numerous simulations have been performed for the values of $R$ reported in Table I, a sinusoidal input $u(t)=3 \sin (2 t)$, initial condition $x(0)=[0,0]^{\top}$ for the plant, $\hat{x}(0)=[5,-5]^{\top}$ for the observer and $\sigma(0)=5$ for the adaptation dynamics. The output measurement $y$ is corrupted by noise generated by the Simulink block Uniform Random Number with sampling time and output range equal to $t_{\mathrm{s}}=0.001 \mathrm{~s}$, and \pm 1 . During the simulation, the noise starts at time $t_{\text {start }}=10$ and ends at $t_{\text {end }}=20$.

A comparison among the true and the estimated state for the two observers $(R=7.5)$ is shown in Figure 1 . Figure 2 shows the estimation error, where the benefit of the dead-zone mechanism can be better appreciated, and the adaptation of the dead-zone level $\sqrt{\sigma}$.

To compare the two observers we propose a tracking Error to Noise Ratio (ENR) which is inspired by the classical concept of Signal to Noise Ratio (SNR). The ENR is formally defined as ENR $:=\frac{\mathbb{E}\left\{e^{\top} e\right\}}{\mathbb{E}\left\{v^{\top} v\right\}}$, where $\mathbb{E}\{\cdot\}$ denotes the expectation operator. The value of the expectations has been obtained simply computing the average of the signals over the interval $\left[t_{\text {start }}, t_{\text {end }}\right]$. The ENRs for the two observers, and for different values of $R$, are reported in the Table I. We can notice that the dead-zone mechanism reduces the tracking error to noise ratio (ENR) and that this reduction is more consistent when the value of $R$ is large. 

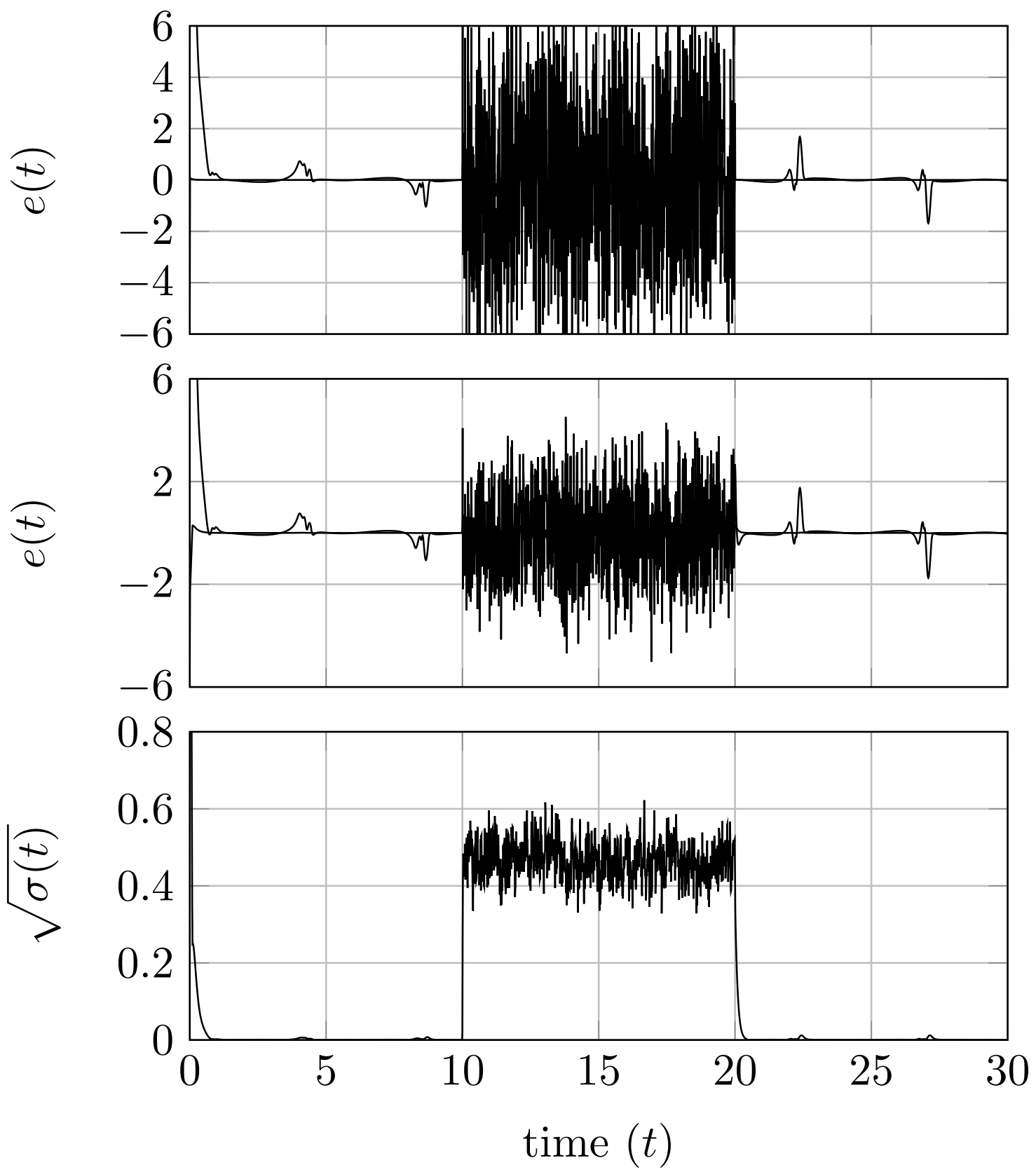

Figure 2. Estimation error for the high-gain observer (top), dead-zonated high-gain observer (middle) $(R=7.5)$, and adaptation of the $\sigma$ level (bottom).

\section{CONCLUSION}

We proposed a dead-zonated output injection term that robustifies Luenberger and high-gain observers against high frequency noise. We proved that the resulting dead-zone observers are not restrictive for LTI plants and can always be designed under standard (and necessary) detectability assumption. Numerical studies within the nonlinear high-gain setting clearly reveal potential to reduce the ISS gain from the measurement noise and the estimation error. The quantitative characterization of this ISS gain appears to be a nontrivial task due to the convoluted nonlinear effect of the dynamic dead-zone, and it is seen as a future work.

\section{REFERENCES}

[1] D. G. Luenberger, "Observing the state of a linear system," IEEE transactions on military electronics, vol. 8, no. 2, pp. 74-80, 1964.

[2] —_, "Observers for multivariable systems," IEEE Transactions on Automatic Control, vol. 11, no. 2, pp. 190-197, 1966.

[3] J. Doyle and G. Stein, "Robustness with observers," IEEE Transactions on Automatic Control, vol. 24, no. 4, pp. 607-611, Aug 1979.

[4] J.-P. Gauthier, H. Hammouri, and S. Othman, "A simple observer for nonlinear systems applications to bioreactors," IEEE Transactions on automatic control, vol. 37, no. 6, pp. 875-880, 1992. 
[5] F. Esfandiari and H. K. Khalil, "Output feedback stabilization of fully linearizable systems," International Journal of control, vol. 56, no. 5, pp. 1007-1037, 1992.

[6] A. R. Teel and L. Praly, "Tools for semiglobal stabilization by partial state and output feedback," SIAM Journal on Control and Optimization, vol. 33, no. 5 , pp. $1443-1488,1995$.

[7] — , "Global stabilizability and observability imply semi-global stabilizability by output feedback," Systems \& Control Letters, vol. 22, no. 5, pp. 313-325, 1994.

[8] A. N. Atassi and H. K. Khalil, "A separation principle for the stabilization of a class of nonlinear systems," IEEE Transactions on Automatic Control, vol. 44, no. 9, pp. 1672-1687, 1999.

[9] H. Kwakernaak and R. Sivan, Linear optimal control systems. Wiley-Interscience New York, 1972, vol. 1.

[10] L. K. Vasiljevic and H. K. Khalil, "Error bounds in differentiation of noisy signals by high-gain observers," Systems \& Control Letters, vol. 57, no. 10, pp. 856-862, 2008.

[11] A. A. Prasov and H. K. Khalil, "Tracking performance of a high-gain observer in the presence of measurement noise," International Journal of Adaptive Control and Signal Processing, vol. 30, no. 8-10, pp. 1228-1243, 2016.

[12] D. Astolfi, L. Marconi, L. Praly, and A. Teel, "Sensitivity to high-frequency measurement noise of nonlinear high-gain observers," in 10th IFAC Symposium on Nonlinear Control Systems, Monterey, CA, 2016, pp. 276-278.

[13] D. Q. Mayne, R. Grainger, and G. C. Goodwin, "Nonlinear filters for linear signal models," IEE Proceedings-Control Theory and Applications, vol. 144, no. 4, pp. 281-286, 1997.

[14] M. S. Chong, D. Nešić, R. Postoyan, and L. Kuhlmann, "Parameter and state estimation of nonlinear systems using a multi-observer under the supervisory framework," IEEE Transactions on Automatic Control, vol. 60, no. 9, pp. 2336-2349, 2015.

[15] J. H. Ahrens and H. K. Khalil, "High-gain observers in the presence of measurement noise: A switched-gain approach," Automatica, vol. 45, no. 4, pp. 936-943, 2009

[16] A. A. Prasov and H. K. Khalil, "A nonlinear high-gain observer for systems with measurement noise in a feedback control framework," IEEE Transactions on Automatic Control, vol. 58, no. 3, pp. 569-580, 2013

[17] R. G. Sanfelice and L. Praly, "On the performance of high-gain observers with gain adaptation under measurement noise," Automatica, vol. 47, no. 10, pp. 2165-2176, 2011.

[18] A. Alessandri and A. Rossi, "Increasing-gain observers for nonlinear systems: Stability and design," Automatica, vol. 57, pp. 180 - $188,2015$.

[19] N. Boizot, E. Busvelle, and J.-P. Gauthier, "An adaptive high-gain observer for nonlinear systems," Automatica, vol. 46, no. 9, pp. 1483-1488, 2010.

[20] V. Andrieu, L. Praly, and A. Astolfi, "Homogeneous approximation, recursive observer design, and output feedback," SIAM Journal on Control and Optimization, vol. 47, no. 4, pp. 1814-1850, 2008.

[21] — "High gain observers with updated gain and homogeneous correction terms," Automatica, vol. 45, no. 2, pp. 422-428, 2009.

[22] D. Astolfi, A. Alessandri, and L. Zaccarian, "Stubborn iss redesign for nonlinear high-gain observers," IFAC-PapersOnLine, vol. 50, no. 1, pp. 15 422$15427,2017$.

[23] D. Astolfi and L. Marconi, "A high-gain nonlinear observer with limited gain power," IEEE Transactions on Automatic Control, vol. 60, no. 11, pp. 3059-3064, 2015

[24] D. Astolfi, L. Marconi, and A. R. Teel, "Low-power peaking-free high-gain observers for nonlinear systems," in Control Conference (ECC), 2016 European. IEEE, 2016, pp. 1424-1429.

[25] J. G. Da Silva and S. Tarbouriech, "Antiwindup design with guaranteed regions of stability: an lmi-based approach," IEEE Transactions on Automatic Control, vol. 50, no. 1, pp. 106-111, 2005.

[26] T. Hu, Z. Lin, and B. M. Chen, "An analysis and design method for linear systems subject to actuator saturation and disturbance," Automatica, vol. 38, no. 2 , pp. 351-359, 2002

[27] A. Tilli and M. Montanari, "A low-noise estimator of angular speed and acceleration from shaft encoder measurement," Automatika-Zagreb, vol. 42, no. 3/4, pp. 169-176, 2001.

[28] A. Alessandri and L. Zaccarian, "Stubborn state observers for linear time-invariant systems," Automatica, vol. 88, pp. 1-9, feb 2018.

[29] M. Cocetti, S. Tarbouriech, and L. Zaccarian, "On dead-zone observers for linear plants," in American Control Conference (ACC). IEEE, June 2018.

[30] H. Shim, J. H. Seo, and A. R. Teel, "Nonlinear observer design via passivation of error dynamics," Automatica, vol. 39, no. 5, pp. 885 - 892, 2003.

[31] J. P. Hespanha, Linear systems theory. Princeton university press, 2009.

[32] J.-P. Gauthier and I. Kupka, Deterministic observation theory and applications. Cambridge university press, 2001.

[33] H. K. Khalil and L. Praly, "High-gain observers in nonlinear feedback control," International Journal of Robust and Nonlinear Control, vol. 24, no. 6, pp. 993-1015, 2014.

[34] P. Bernard, L. Praly, and V. Andrieu, "Observers for a non-lipschitz triangular form," Automatica, vol. 82, pp. 301-313, 2017. 